\title{
Characterizing the mechanism(s) of heavy element synthesis reactions
}

\author{
Walter Loveland ${ }^{\text {a }}$ \\ Department of Chemistry, Oregon State University, Corvallis, OR 97331, USA
}

\begin{abstract}
A review of the current state of our understanding of complete fusion reaction mechanisms is presented, from the perspective of an experimentalist. For complete fusion reactions, the overall uncertainties in predicting heavy element synthesis cross sections are examined in terms of the uncertainties associated with the calculations of capture cross sections, fusion probabilities and survival probabilities.
\end{abstract}

\section{Introduction}

Formally, the cross section for producing a heavy evaporation residue, $\sigma_{\mathrm{EVR}}$, in a fusion reaction can be written as

$$
\sigma_{\mathrm{EVR}}(E)=\frac{\pi h^{2}}{2 \mu E} \sum_{\ell=0}^{\infty}(2 \ell+1) T(E, \ell) P_{\mathrm{CN}}(E, \ell) W_{\text {sur }}(E, \ell)
$$

where $E$ is the center of mass energy, and $T$ is the probability of the colliding nuclei to overcome the potential barrier in the entrance channel and reach the contact point. (The term "evaporation residue" refers to the product of a fusion reaction followed by the evaporation of a specific number of neutrons.) $P_{\mathrm{CN}}$ is the probability that the projectile-target system will evolve from the contact point to the compound nucleus. $W_{\text {sur }}$ is the probability that the compound nucleus will decay to produce an evaporation residue rather than fissioning. Conventionally the EVR cross section is separated into three individual reaction stages (capture, fusion, survival) motivated, in part, by the different time scales of the processes. However, one must remember that the $W_{\text {sur }}$ term effectively sets the allowed values of the spin. This effect is shown in Fig. 1 where the capture cross sections for several reactions are shown without and with the spin limitation posed by the survival probabilities.

Several successful attempts have been made to describe the cross sections for evaporation residue formation in cold fusion reactions [2-5]. In Fig. 2a, I show some typical examples of post dictions of the formation cross sections for elements 104-113 in cold fusion reactions. The agreement between theory and experiment is impressive because the cross sections extend over six orders of magnitude, i.e., a robust agreement. Because the values of $\sigma_{\text {capture }}$ are well known or generally agreed upon (see below), the values of the product $P_{\mathrm{CN}} \cdot W_{\text {sur }}$ are the same in most of these post dictions. However, as seen in Fig. 2b, the values of $P_{\mathrm{CN}}$ differ significantly in these post dictions [2-5], and differ from measurements of $P_{\mathrm{CN}}$ [6]. A similar situation occurs in predictions of cross sections for hot fusion reactions. These are clear-cut cases in which a simple agreement between theory and experiment in postdicted cross sections is not sufficient to indicate a real understanding of the phenomena involved.

\footnotetext{
${ }^{a}$ e-mail: lovelanw@onid.orst.edu

(C) The Authors, published by EDP Sciences. This is an Open Access article distributed under the terms of the Creative Commons Attribution License 4.0 (http://creativecommons.org/licenses/by/4.0/).
} 

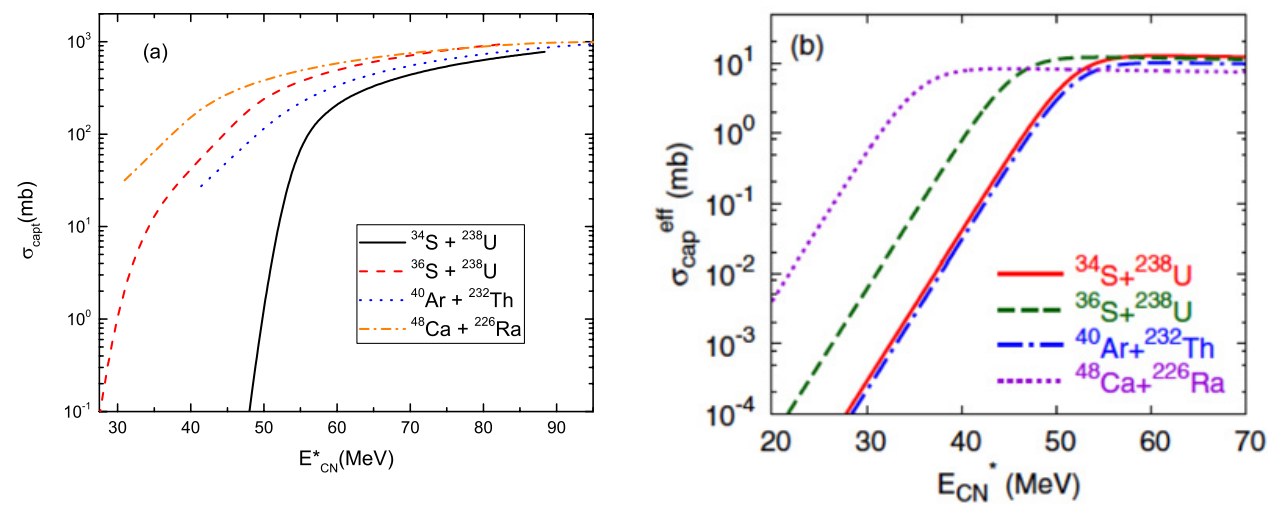

Figure 1. (a) Calculated capture cross sections for some typical reactions. (b) the "spin-limited" capture cross sections for the reactions in (a) [1].
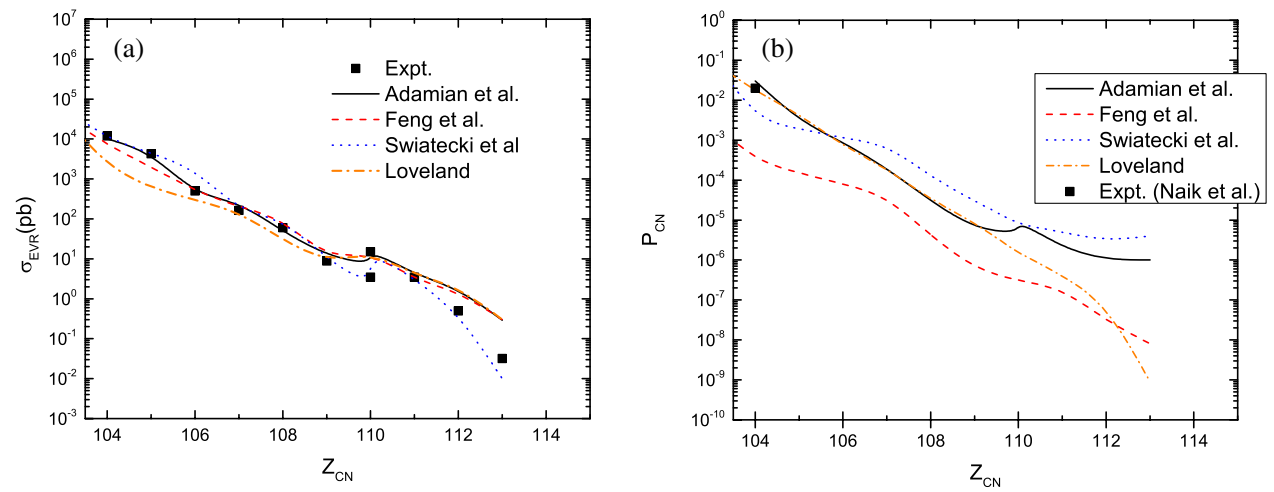

Figure 2. (a) Typical predictions of the formation cross sections of elements 104-113 using cold fusion reactions. (b) $P_{\mathrm{CN}}$ values for the predictions in panel (a). The references cited in the legends refer to Adamian [4], Feng [5], Swiatecki [2], and Loveland [3]. The additional reference in panel (b) is to the data of Naik et al. [6].

We might ask what the overall uncertainties are in the current phenomenological models for predicting heavy element production cross sections. This is an item of some controversy. Some feel the uncertainties in typical predictions are factors of 2-4 [7] while others estimate these uncertainties to be $1-2$ orders of magnitude $[8,9]$.

\section{Capture cross sections}

The capture cross section is, in the language of coupled channel calculations, the "barrier crossing" cross section. It is the sum of the quasifission, fast fission, fusion-fission and fusionevaporation residue cross sections. The barriers involved are the interaction barriers and not the fusion barriers. There are several models for capture cross sections. Each of them has been tested against a number of measurements of capture cross sections for reactions that, mostly, do not lead to the formation of the heaviest nuclei. In general, these models are able to describe the magnitudes of the capture cross sections within 50\% and the values of the interaction barriers within $20 \%$. The most robust of these models takes into account the effects of target and projectile orientation/deformation upon the collisions, the couplings 

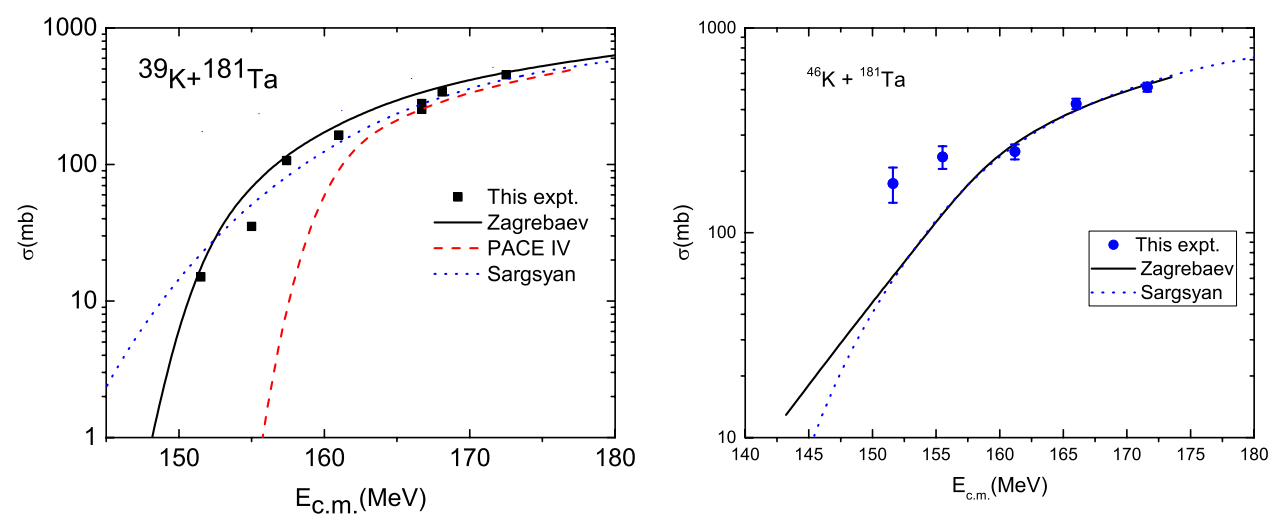

Figure 3. The capture-fission excitation functions for the reaction of ${ }^{39} \mathrm{~K}$ (left) and ${ }^{46} \mathrm{~K}$ (right) with ${ }^{181} \mathrm{Ta}$.

associated with inelastic excitations of the target and projectile and the possibility of one or two neutron transfer processes. Loveland [10] has compared calculations of the capture cross sections for reactions that synthesize heavy nuclei with the measured cross sections. Good agreement between the measured and calculated values of the cross sections occurs for all reactions. The ratio of calculated to observed capture cross sections varies from 0.5 to 2 . Nominally, given the other uncertainties in estimating $\sigma_{\mathrm{EVR}}$, this seems generally acceptable. However, from the point of view of an experimentalist, it is not acceptable. The capture cross section is relatively easy to measure and an uncertainty of a factor of 50\% may mean having to run an experiment for several months longer to be successful in a synthetic effort.

Future synthetic efforts with heavy nuclei may involve the use of very neutron-rich beams such as radioactive beams. While we understand that such efforts are not likely to produce new superheavy elements due to the low intensities of the radioactive beams [3], there may exist a window of opportunity to make new neutron-rich isotopes of elements 104-108 [11]. Our ability to predict the capture cross sections for the interaction of very neutron-rich projectiles with heavy nuclei is limited [11] and this is especially true near the interaction barrier where the predictions of models of the capture cross sections may differ by orders of magnitude [11]. As part of the effort to study the scientific issues that will be relevant at next generation radioactive beam facilities, such as FRIB, we have started to use the ReA3 facility at the NSCL to study capture processes with fast-releasing beams such as the potassium isotopes. We chose to study the interaction of ${ }^{39,46} \mathrm{~K}$ with ${ }^{181} \mathrm{Ta}$. The first preliminary results from that experiment are shown in Fig. 3. The ${ }^{39} \mathrm{~K}+{ }^{181}$ Ta reaction results seem to be well understood within conventional pictures of capture $[12,13]$ while the neutron-rich ${ }^{46} \mathrm{~K}$ results suggest an unusual near barrier fusion enhancement.

\section{Survival probabilities, $W_{\text {sur }}$}

Formally $W_{\text {sur }}$ can be written as

$$
W_{\text {sur }}\left(E_{\mathrm{c} . \mathrm{m} .}\right)=P_{x n}\left(E_{\mathrm{CN}}^{*}\right) \prod_{i=1}^{x} \frac{\Gamma_{n}\left(E_{i}^{*}\right)}{\Gamma_{n}\left(E_{i}^{*}\right)+\Gamma_{f}\left(E_{i}^{*}\right)}
$$

where $P_{x n}$ is the probability of emitting $x$ (and only $x$ ) neutrons from a nucleus with excitation energy $E^{*}$, and $\Gamma_{n}$ and $\Gamma_{f}$ are the partial widths for decay of the completely fused system by either neutron emission or fission, respectively. For the most part, the formalism for 
calculating the survival, against fission, of a highly excited nucleus is understood. There are significant uncertainties, however, in the input parameters for these calculations and care must be used in treating some situations. "Kramers effects" and the overall fission barrier height are found [14] to have the biggest effect on the calculated cross sections.

A recent experiment concerning survival probabilities in hot fusion reactions showed the importance of "Kramers effects" [15]. The nucleus ${ }^{274} \mathrm{Hs}$ was formed at an excitation energy of $63 \mathrm{MeV}$ using the ${ }^{26} \mathrm{Mg}+{ }^{248} \mathrm{Cm}$ reaction. ${ }^{274} \mathrm{Hs}$ has several interesting properties. The liquid drop model fission barrier height is zero and there is a subshell at $N=162, Z=108$. In the formation reaction, $P_{\mathrm{CN}}$ is measured [16] to be 1.0. By measuring the angular distribution of the fission associated neutrons, Yanez et al. [15] were able to deduce a value of $\Gamma_{n} / \Gamma_{\text {total }}$ for the first chance fission of ${ }^{274} \mathrm{Hs}\left(E^{*}=63 \mathrm{MeV}\right)$ of $0.89 \pm 0.13$ ! A highly excited fragile nucleus with a vanishingly small fission barrier decayed $\sim 90 \%$ of the time by emitting a neutron rather than fissioning. Conventional calculations with various values of the fission barrier height were unable to reproduce these results. The answer to this dilemma is to consider the effects of nuclear viscosity to retard fission [17], the so-called Kramers effects. These Kramers effects are the reason that hot fusion reactions are useful in heavy element synthesis, in that the initial high excitation energies of the completely fused nuclei do not result in catastrophic losses of surviving nuclei [18].

With respect to fission barrier heights, most modern models do equally well/poorly in describing fission barrier heights for Th-Cf nuclei. Afanasjev et al. [19] found the average deviation between the calculated and known inner barrier heights was $\sim 0.7 \mathrm{MeV}$ amongst various models. Bertsch et al. [20] estimate the uncertainties in fission barrier heights are $0.5-1.0 \mathrm{MeV}$ in known nuclei. Kowal et al. [21] found for even-even nuclei with $Z=92-98$ the difference between measured and calculated inner barrier heights was $0.8 \mathrm{MeV}$. Baran et al. [22] found very large, i.e., several $\mathrm{MeV}$, differences between various calculated fission barrier heights for $Z=112-120$. In summary, fission barrier heights are known within $0.5-1.0 \mathrm{MeV}$. For super-heavy nuclei, the change of fission barrier height by $1 \mathrm{MeV}$ in each neutron evaporation step can cause an order of magnitude uncertainty in the $4 n$-channel. For the $3 n$-channel, the uncertainty is about a factor of four [14].

An additional problem is that at the high excitation energies characteristic of hot fusion reactions, the shell effects stabilizing the fission barrier are predicted [23] to be "washed out" with a resulting fission barrier height $<1 \mathrm{MeV}$ for some cases. Furthermore the rate of damping of the shell effects differs from nucleus to nucleus. This point is well illustrated by the calculations in reference [24] of the "effective" fission barrier heights for the ${ }^{48} \mathrm{Ca}+{ }^{249} \mathrm{Bk}$ reaction.

Measurements of fission barrier heights are difficult and the results depend on the models used in the data analysis. Recently Hofmann et al. [25] have deduced the shell-correction energies from the systematics of the $Q_{\alpha}$ values for the heaviest nuclei and used these shellcorrection energies to deduce fission barrier heights. The deduced barrier heights for elements 118 and 120 may be larger than expected.

\section{Fusion probability, $\boldsymbol{P}_{\mathrm{CN}}$}

The fusion probability, $P_{\mathrm{CN}}$, is the least known (experimentally) of the factors affecting complete fusion reactions and perhaps the most difficult to model. The essential task is to measure the relative amounts of fusion-fission and quasifission in a given reaction. Experimentally this is done using mass-angle correlations where it is difficult to measure, with any certainty, the fraction of fusion reactions when that quantity is less than 1\%. (For cold fusion reactions, $P_{\mathrm{CN}}$ is predicted to take on values of $10^{-2}$ to $10^{-6}$ for reactions that make elements 
104-113.) The reaction of ${ }^{124} \mathrm{Sn}$ with ${ }^{96} \mathrm{Zr}$ can be used to illustrate the uncertainties in the theory to estimate $P_{\mathrm{CN}}$ where [10] various theoretical estimates of $P_{\mathrm{CN}}$ range from 0.0002 to 0.56 and where the measured value is 0.05 as well as the data shown in Fig. $2 b$.

Where we have made progress in understanding $P_{\mathrm{CN}}$ is in the excitation energy dependence of $P_{\mathrm{CN}}$. Zagrebaev and Greiner [26] have suggested the following ad hoc functional form for the excitation energy dependence of $P_{\mathrm{CN}}$

$$
P_{\mathrm{CN}}\left(E^{*}, J\right)=\frac{P_{\mathrm{CN}}^{0}}{1+\exp \left[\frac{E_{B}^{*}-E_{\mathrm{int}}^{*}(J)}{\Delta}\right]}
$$

where $P_{\mathrm{CN}}^{0}$ is the fissility-dependent "asymptotic" (above barrier) value of $P_{\mathrm{CN}}$ at high excitation energies, $E_{B}^{*}$ is the excitation energy at the Bass barrier, $E_{\mathrm{int}}^{*}(J)$ is the internal excitation energy $\left[E_{c . m}+Q-E_{\mathrm{rot}}(J)\right], J$ is the angular momentum of the compound nucleus, and $\Delta$ (an adjustable parameter) is taken to be $4 \mathrm{MeV}$. This formula describes the extensive data of Knyazheva et al. [27] for the ${ }^{48} \mathrm{Ca}+{ }^{154} \mathrm{Sm}$ reaction very well [10]. A generalization of this formula has been used to describe the excitation energy dependence of $P_{\mathrm{CN}}$ for the reactions of ${ }^{48} \mathrm{Ca}$ with ${ }^{238} \mathrm{U},{ }^{244} \mathrm{Pu}$, and ${ }^{248} \mathrm{Cm}[28]$.

It is also clear that $P_{\mathrm{CN}}$ must depend on the entrance channel asymmetry of the reaction. Numerous scaling factors to express this dependence have been proposed and used. An extensive survey of $P_{\mathrm{CN}}$ in a large number of fusing systems was made by du Rietz et al. [29]. They thought that perhaps some fissility related parameter would be the best way to organize their data on $P_{\mathrm{CN}}$ and its dependence of the properties of the entrance channel in the reactions they studied. They found the best fissility-related scaling variable that organized their data was $x_{\text {duRietz }}=0.75 x_{\text {eff }}+0.25 x_{\mathrm{CN}}$. The parameters $x_{\text {eff }}$ and $x_{\mathrm{CN}}$ are the associated fissilities for the entrance channel and the compound system, respectively. The following equations can be used to calculate these quantities

$$
\begin{aligned}
x_{\mathrm{CN}} & =\frac{\left(Z^{2} / A\right)_{\mathrm{CN}}}{\left(Z^{2} / A\right)_{\text {critical }}} ; \quad\left(Z^{2} / A\right)_{\text {critical }}=50.883 \cdot\left[1 .-1.7826\left(\frac{A-2 Z}{A}\right)^{2}\right] ; \\
x_{\text {eff }} & =\frac{4 Z_{1} Z_{2} /\left[A_{1}^{1 / 3} A_{2}^{1 / 3}\left(A_{1}^{1 / 3}+A_{2}^{1 / 3}\right)\right]}{\left(Z^{2} / A\right)_{\text {critical }}} .
\end{aligned}
$$

In Fig. 4, I show most of the known data on $P_{\mathrm{CN}}$ using the du Rietz scaling variable. There is no discernable pattern. Restricting the choice of cases to those in a narrow excitation energy bin improves the situation somewhat but it is clear we are missing something in our semiempirical systematics.

Some progress has been made in calculating $P_{\mathrm{CN}}$ using TDHF calculations. Wakhle et al. [30] made a pioneering study of the ${ }^{40} \mathrm{Ca}+{ }^{238} \mathrm{U}$ reaction. The capture cross sections predicted by their TDHF calculations agreed with measured capture cross sections [31] within $\pm 20 \%$. In addition they were able to predict the ratio of fusion to capture cross sections, $\sigma_{\text {fus }} / \sigma_{\text {capture }}$, as $0.09 \pm 0.07$ at $205.9 \mathrm{MeV}$ and $0.16 \pm 0.06$ at $225.4 \mathrm{MeV}$ in agreement with reference [31] who measured ratios of $0.06 \pm 0.03$ and $0.14 \pm 0.05$, respectively. Whether TDHF calculations will become a predictive tool for heavy element synthesis remains to be seen.

\section{Predictions for the production of elements 119 and 120}

Loveland [10] has shown that the current predictions for the production cross sections for elements 119 and 120 differ by 1-3 orders of magnitude, reflecting the uncertainties discussed above. For the reaction ${ }^{50} \mathrm{Ti}+{ }^{249} \mathrm{Bk} \rightarrow 119$, the uncertainties in the predicted maximum 

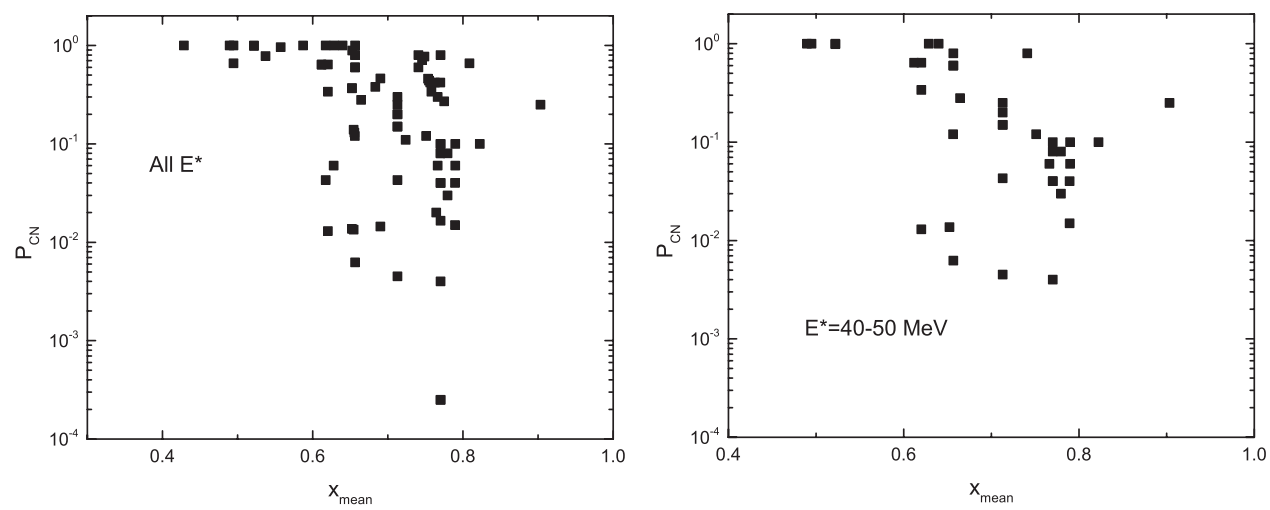

Figure 4. Fissility dependence of $P_{\mathrm{CN}}$ with and without excitation energy sorting.

cross sections for the $3 n$ and $4 n$ channels differ by "only" a factor of 20-40 while larger uncertainties are found in the predictions for the ${ }^{54} \mathrm{Cr}+{ }^{248} \mathrm{Cm} \rightarrow 120$ reaction. The energies of the maxima of the $3 n$ and $4 n$ excitation functions are uncertain to $3-4 \mathrm{MeV}$, a troublesome situation for the experimentalist.

\section{Conclusions}

I conclude that: (a) capture cross sections should be measured for reactions of interest. (b) We need better and more information on fission barrier heights, and their changes with excitation energy for the heaviest nuclei. (c) We need to devise better methods of measuring $P_{\mathrm{CN}}$ and more TDHF calculations of $P_{\mathrm{CN}}$. (d) The current uncertainty in calculated values of $\sigma_{\mathrm{EVR}}$ is at least 1-2 orders of magnitude. (e) New opportunities for making neutron-rich actinides with RNBs may exist.

This work was supported, in part, by the Director, Office of Energy Research, Division of Nuclear Physics of the Office of High Energy and Nuclear Physics of the U.S. Department of Energy under Grant DE-SC0014380 and the National Science Foundation under award 1505043.

\section{References}

[1] J. Hong, G.G. Adamian, N.V. Antonenko, Phys. Rev. C 92, 014617 (2015).

[2] W.J. Swiatecki, K. Siwek-Wilczynska, J. Wilczynski, Phys.Rev. C 71, 014602 (2005).

[3] W. Loveland, Phys. Rev. C 76, 014612 (2007).

[4] G.G. Adamian, N.V. Antonenko, W. Scheid, Nucl. Phys. A678, 24 (2000).

[5] Z.-Q. Feng, G.-M. Jin, J.-Q. Li, W. Scheid, Phys. Rev. C 76, 044606 (2007).

[6] R.S. Naik et al., Phys. Rev. C 76, 054604 (2007).

[7] G.G. Adamian, N.V. Antonenko, W. Scheid, Phys. Rev. C 69, 014607 (2004).

[8] V.I. Zagrebaev, Y. Aritomo, M.G. Itkis, Y.T. Oganessian, M. Ohta, Phys. Rev. C 65, 014607 (2001).

[9] V.I. Zagrebaev and W. Greiner, Nucl. Phys. A944, 257 (2015).

[10] W. Loveland, Eur. Phys. J. A51, 120 (2015).

[11] W. Loveland, J. Phys. Conf. Series 420, 012004 (2013).

[12] http://nrv.jinr.ru/nrv/ 
[13] V.G. Sargsyan (private communication).

[14] H. Lu, D. Boilley, EPJ Web of Conferences 62, 03002 (2013).

[15] R. Yanez et al., Phys. Rev. Lett. 112, 152702 (2014).

[16] M.G. Itkis et al., AIP Conf. Proc. 853, 231 (2006).

[17] H.A. Kramers, Physica 7, 284 (1940).

[18] A.N. Andreyev et al., Heavy Ion Fusion: Exploring the Variety of Nuclear Properties (Singapore, World Scientific, 1994), 260.

[19] A.V. Afanasjev, H. Abushara, P. Ring, Int. J. Mod. Phys. E 21, 1250025 (2012).

[20] G. Bertsch, W. Loveland, W. Nazarewicz, P. Talou, J. Phys. G 42, 1 (2015).

[21] M. Kowal, P. Jachimowicz, A. Sobiczewski, Phys. Rev. C 82, 014303 (2010).

[22] A. Baran, M. Kowal, P.-G. Reinhard, L.M. Robledo, A. Staszczak, M. Warda, Nucl. Phys. A944, 442 (2015).

[23] J.C. Pei, W. Nazarewicz, J.A. Sheikh, A.K. Kerman, Phys. Rev. Lett. 102, 192501 (2009).

[24] G. Giardina et al., J. Phys. Conf. Series 282, 012006 (2011).

[25] S. Hofmann et al., Eur. Phys. J. A52, 116 (2016).

[26] V. Zagrebaev and W. Greiner, Phys. Rev. C 78, 034610 (2008).

[27] G.N. Knyazheva et al., Phys. Rev. C 75, 064602 (2007).

[28] E.M. Kozulin et al., Phys. Rev. C 90054608 (2014).

[29] R. du Rietz et al., Phys. Rev. C 88, 054618 (2013).

[30] A. Wakhle et al., Phys. Rev. Lett. 113, 182502 (2014).

[31] W.Q. Shen et al., Phys. Rev. C 36, 115 (1987). 